\title{
ON MAKAROV'S PRINCIPLE IN CONFORMAL MAPPING
}

\author{
OLEG IVRII
}

\begin{abstract}
We examine several characteristics of conformal maps that resemble the variance of a Gaussian: asymptotic variance, the constant in Makarov's law of iterated logarithm and the second derivative of the integral means spectrum at the origin. While these quantities need not be equal in general, they agree for domains whose boundaries are regular fractals such as Julia sets or limit sets of quasi-Fuchsian groups. We give a new proof of these dynamical equalities. We also show that these characteristics have the same universal bounds and prove a central limit theorem for extremals. Our method is based on analyzing the local variance of dyadic martingales associated to Bloch functions.
\end{abstract}

\section{INTRODUCTION}

Let $\Omega \subset \mathbb{C}$ be a simply connected domain in the plane whose boundary is a Jordan curve. In 1985, N. Makarov [18] introduced probabilistic techniques into the theory of conformal mapping to show that the harmonic measure on $\partial \Omega$ necessarily has Hausdorff dimension 1. This is quite surprising for domains $\Omega$ with H. $\operatorname{dim} \partial \Omega>1$ : for such domains, Makarov's theorem suggests that Brownian motion started at an interior point $z_{0} \in \Omega$ only hits a small subset of the boundary. Later, Makarov obtained finer estimates for the metric properties of harmonic measure by proving a law of iterated logarithm for Bloch functions. Central to Makarov's reasoning was the following insight: Bloch functions behave like martingales.

1.1. Three characteristics of Bloch functions. By definition, the Bloch space consists of holomorphic functions on the unit disk for which the Bloch norm $\|b\|_{\mathcal{B}}:=\sup _{z \in \mathbb{D}}\left|b^{\prime}(z)\right|\left(1-|z|^{2}\right)$ is finite. Bloch functions naturally arise from conformal mappings: if $f$ is a conformal mapping, then $\left\|\log f^{\prime}\right\|_{\mathcal{B}} \leqslant 6$. While a Bloch function $b \in \mathcal{B}$ can grow like $|b(z)| \sim \log \frac{1}{1-|z|}$, the martingale property of $b$ suggests that the average rate of growth is much smaller -

2010 Mathematics Subject Classification. Primary 30C35; Secondary 30C62.

The author was supported by the Academy of Finland, project nos. 271983 and 273458. 
approximately the square root of the maximum. With this in mind, it is natural to expect that the following three characteristics are finite:

- The asymptotic variance

$$
\sigma^{2}(b)=\limsup _{r \rightarrow 1} \frac{1}{2 \pi|\log (1-r)|} \int_{|z|=r}|b(z)|^{2}|d z| .
$$

- The LIL constant

$$
C_{\mathrm{LIL}}(b)=\underset{\theta \in[0,2 \pi)}{\operatorname{ess} \sup }\left\{\limsup _{r \rightarrow 1} \frac{\left|b\left(r e^{i \theta}\right)\right|}{\sqrt{\log \frac{1}{1-r} \log \log \log \frac{1}{1-r}}}\right\} .
$$

- The integral means spectrum

$$
\beta_{b}(\tau)=\limsup _{r \rightarrow 1} \frac{1}{|\log (1-r)|} \cdot \log \int_{|z|=r}\left|e^{\tau b(z)}\right||d z|, \quad \tau \in \mathbb{C} .
$$

The fact " $C_{\mathrm{LIL}}^{2}(b) \leqslant C_{1}\|b\|_{\mathcal{B}}^{2}$ " is Makarov's celebrated law of iterated logarithm mentioned above. The inequalities $\sigma^{2}(b) \leqslant C_{2}\|b\|_{\mathcal{B}}^{2}$ and $\beta_{b}(\tau) \leqslant$ $C_{3}|\tau|^{2}\|b\|_{\mathcal{B}}^{2}$ are also well known and can be found in Pommerenke's book [24]. We will reprove these facts here with an emphasis on sharp constants.

1.2. Dynamical equalities. In dynamical situations, the above characteristics are linked by an explicit relation:

Theorem 1.1. Suppose $f(z)$ is a conformal map, such that the image of the unit circle $f\left(\mathbb{S}^{1}\right)$ is a Jordan curve, invariant under a hyperbolic conformal dynamical system. Then,

$$
\left.2 \frac{d^{2}}{d \tau^{2}}\right|_{\tau=0} \beta_{\log f^{\prime}}(\tau)=\sigma^{2}\left(\log f^{\prime}\right)=C_{\mathrm{LIL}}^{2}\left(\log f^{\prime}\right)
$$

The equalities in (1.4) are mediated by a fourth quantity involving the dynamical asymptotic variance of a Hölder continuous potential from thermodynamic formalism. Theorem 1.1 has a rich history: the connection with $C_{\mathrm{LIL}}^{2}$ is due to Przytycki, Urbański, Zdunik [25], with integral means due to Makarov and Binder [21, 5], and with $\sigma^{2}$ by McMullen [22]. See also [1] for additional details. One of our central objectives is to give a new proof of Theorem 1.1 that does not involve thermodynamic formalism. Instead, we work with a new central quantity: the local variance of a dyadic martingale associated to a Bloch function. The definition will be given in Section 2 .

We emphasize that the above quantities are unrelated for general Bloch functions. We refer the reader to [3, 17] for interesting examples. 
1.3. Universal bounds. It is natural to ask if the above characteristics agree on the level of universal bounds, taken over all conformal maps. We show that this is essentially the case; however, in order to be able to localize these characteristics, we are forced to restrict to conformal maps that have quasiconformal extensions with bounded distortion.

To be concrete, let $\mathbf{S}$ be the class of conformal maps $f: \mathbb{D} \rightarrow \mathbb{C}$ normalized so that $f(0)=0$ and $f^{\prime}(0)=1$, and for $0<k<1$, let $\mathbf{S}_{k} \subset \mathbf{S}$ denote the collection of maps that admit a $k$-quasiconformal extension to the complex plane. Let $B_{k}(\tau):=\sup _{f \in \mathbf{S}_{k}} \beta_{\log f^{\prime}}(\tau)$.

Theorem 1.2. For any $0<k<1$,

$$
\lim _{\tau \rightarrow 0} \frac{B_{k}(\tau)}{|\tau|^{2} / 4}=\sup _{f \in \mathbf{S}_{k}} \sigma^{2}\left(\log f^{\prime}\right)=\sup _{f \in \mathbf{S}_{k}} C_{\mathrm{LIL}}^{2}\left(\log f^{\prime}\right) .
$$

In fact, we will show that for each of the three expressions, it suffices to take the supremum over fractal objects that satisfy Theorem 1.1. The above quantity will be denoted $\Sigma^{2}(k)$. As discussed in [13], $\Sigma^{2}(k) / k^{2}$ is a non-decreasing convex function of $k$. It is currently known that

$$
0.93<\lim _{k \rightarrow 1^{-}} \Sigma^{2}(k)<(1.24)^{2} .
$$

We refer the reader to [1, Section 8] for the lower bound and to [10, 11, 12 , for the upper bound. A theorem of Makarov [19], [7, Theorem VIII.2.1] shows:

Corollary 1.3. (i) Let $\Omega=f(\mathbb{D})$ be the image of the unit disk and $z_{0}$ be a point in $\Omega$. The harmonic measure $\omega_{z_{0}}$ on $\partial \Omega$, as viewed from $z_{0}$, is absolutely continuous with respect to the Hausdorff measure $\Lambda_{h(t)}$,

$$
h(t)=t \exp \left\{C \sqrt{\log \frac{1}{t} \log \log \log \frac{1}{t}}\right\}, \quad 0<t<10^{-7},
$$

for any $C \geqslant C_{\mathrm{LIL}}\left(b_{f}\right)$. In particular, $C=\sqrt{\Sigma^{2}(k)}$ works.

(ii) Conversely, if $C<\sqrt{\Sigma^{2}(k)}$, there exists a conformal map $f \in \mathbf{S}_{k}$ for which $\omega_{z_{0}} \perp \Lambda_{h(t)}$.

The connections to LIL in Theorem 1.2 and Corollary 1.3 were originally proved together with I. Kayumov using a different method than presented here. 
In Section 6, we will show that extremal Bloch functions obey a central limit theorem. For a fixed $r<1$, we may consider

$$
\tilde{b}_{r}(\theta):=\frac{b\left(r e^{i \theta}\right)}{\sqrt{|\log (1-r)|}}
$$

as a random variable with respect to the probability measure $|d z| / 2 \pi$.

Theorem 1.4. Let $0<k<1$ be fixed. Suppose $f \in \mathbf{S}_{k}$ and $b=\log f^{\prime}$. Given $\varepsilon>0$, there exists a $\delta>0$ such that if $r$ is sufficiently close to 1 and

$$
\sigma^{2}(b, r)=\frac{1}{2 \pi|\log (1-r)|} \int_{|z|=r}|b(z)|^{2}|d z|>\Sigma^{2}(k)-\delta,
$$

then the distribution of $\tilde{b}_{r}$ is close to a complex Gaussian of mean 0 and variance $\Sigma^{2}(k)$, up to an additive error of at most $\varepsilon$. In other words, $\operatorname{Re} \tilde{b}_{r}$ and $\operatorname{Im} \tilde{b}_{r}$ approximate independent real Gaussians of variance $\Sigma^{2}(k) / 2$.

Loosely speaking, extremality forces fractal structure.

1.4. Infinitesimal analogue. Suppose $\mu \in M(\mathbb{D})$ is a Beltrami coefficient on the unit disk with $\|\mu\|_{\infty} \leqslant 1$. For $t \in \mathbb{D}$, let $w^{t \mu}$ be the principal solution to the Beltrami equation $\bar{\partial} w=t \mu \partial w$. By construction, $\varphi_{t}=\left.w^{t \mu}\right|_{\mathbb{D}^{*}}$ is a holomorphic family of conformal mappings of the exterior unit disk, with $\varphi_{0}$ being the identity map. In this setting, $t \rightarrow \log \varphi_{t}^{\prime}$ is a Banach-valued holomorphic function (to the Bloch space of the exterior unit disk) whose derivative at the origin is the Beurling transform

$$
\mathcal{S} \mu(z)=-\frac{1}{\pi} \int_{\mathbb{D}} \frac{\mu(w)}{(z-w)^{2}}|d w|^{2}, \quad|z|>1
$$

i.e.

$$
\left\|\log \left(w^{t \mu}\right)^{\prime}-t \mathcal{S} \mu\right\|_{\mathcal{B}\left(\mathbb{D}^{*}\right)}=\mathcal{O}\left(|t|^{2}\right), \quad t \in \mathbb{D} .
$$

We refer the reader to [13, Section 2] for details. Consider the rescaled integral means spectrum

$$
B_{*}(\tau):=\lim _{k \rightarrow 0} B_{k}(\tau / k)=\sup _{|\mu| \leqslant \chi_{\mathbb{D}}} \beta_{\mathcal{S} \mu}(\tau) .
$$

Since the collection of Bloch functions $\left\{\mathcal{S} \mu,|\mu| \leqslant \chi_{\mathbb{D}}\right\}$ is invariant under rotation by $e^{i \theta} \in \mathbb{S}^{1}, B_{*}(\tau)$ only depends on $|\tau|$. Whether or not $B_{k}(\tau)$ is radially-invariant is an open problem. We have: 


\section{Corollary 1.5.}

$$
\lim _{\tau \rightarrow 0} \frac{B_{*}(\tau)}{|\tau|^{2} / 4}=\sup _{|\mu| \leqslant \chi_{\mathbb{D}}} \sigma^{2}(\mathcal{S} \mu)=\sup _{|\mu| \leqslant \chi_{\mathbb{D}}} C_{\mathrm{LIL}}^{2}(\mathcal{S} \mu)
$$

We denote the common quantity by $\Sigma^{2}$. From the above construction, it is clear that $\Sigma^{2}=\lim _{k \rightarrow 0} \Sigma^{2}(k) / k^{2}$. The quantity $\Sigma^{2}$ was first studied in [1], where it was established that $0.87913 \leqslant \Sigma^{2} \leqslant 1$, while Hedenmalm [9] proved the strict inequality $\Sigma^{2}<1$.

1.5. Notes and references. In [1], the original motivation for investigating $\Sigma^{2}$ arose from the question of finding $D(k)$, the maximal Minkowski dimension of a $k$-quasicircle. The anti-symmetrization procedure of [16, 26] and the relation [24, Corollary 10.18]

$$
\beta_{f}(t)=t-1 \Longleftrightarrow t=\mathrm{M} \cdot \operatorname{dim} f\left(\mathbb{S}^{1}\right), \quad f \in \mathbf{S}_{k},
$$

reduce the problem of finding $D(k)$ to investigating the function $B_{k}(\tau)$. In [13], the author modified the argument of Becker and Pommerenke [4] for estimating integral means to show the asymptotic expansion

$$
D(k)=1+\Sigma^{2} k^{2}+\mathcal{O}\left(k^{8 / 3-\varepsilon}\right) .
$$

Together with Hedenmalm's estimate $\Sigma^{2}<1$, this improves on Smirnov's bound $D(k) \leqslant 1+k^{2}$ from [26]. In this paper, we use Makarov's Bloch martingale technique to give an alternative perspective on the results from [13. In Section 5, we give an estimate for $B_{*}(\tau)$ which implies 1.10 , albeit with a slightly weaker error term.

An a priori difficulty in studying $\Sigma^{2}$ is that the extremal problem of maximizing asymptotic variance $\sup _{|\mu| \leqslant \chi \mathbb{D}} \sigma^{2}(\mathcal{S} \mu)$ has infinitely many solutions. For instance, one can take an extremal $\mu$ and modify it in an arbitrary manner on a compact subset of the disk. Alternatively, one can pullback an extremal $\mu(z) \frac{d \bar{z}}{d z}$ by a Blaschke product $B: \mathbb{D} \rightarrow \mathbb{D}$. Further, given two extremals $\mu, \nu$, one can glue them together $\mu \cdot\left\{\chi_{\operatorname{Re} z<0}\right\}+\nu \cdot\left\{\chi_{\operatorname{Re} z>0}\right\}$ to form yet another extremal. These facts follow from the localization principle, see [13, Section 4].

In [1, Section 6], extremal Bloch functions were studied indirectly via fractal approximation. The idea was to show

$$
\Sigma^{2}=\sup _{\mu \in M_{\mathrm{I}},|\mu| \leqslant \chi_{\mathbb{D}}} \sigma^{2}(\mathcal{S} \mu)
$$


where $M_{\mathrm{I}}$ is the class of Beltrami coefficients that are eventually-invariant under $z \rightarrow z^{d}$ for some $d \geqslant 2$, i.e. satisfying $\left(z^{d}\right)^{*} \mu=\mu$ in some open neighbourhood of the unit circle. There, the fractal approximation technique was used to deduce $\Sigma^{2} \leqslant 1$ from Smirnov's $1+k^{2}$ bound.

Surprisingly, fractal approximation fails in the Fuchsian setting:

$$
\Sigma_{\mathrm{F}}^{2}=\sup _{\mu \in M_{\mathrm{F}},|\mu| \leqslant \chi \mathbb{D}} \sigma^{2}(\mathcal{S} \mu)<2 / 3<\Sigma^{2}
$$

where $M_{\mathrm{F}}$ is the class of Beltrami coefficients invariant under some cocompact Fuchsian group. By the work of McMullen [22], the problem of understanding $\Sigma_{\mathrm{F}}^{2}$ is naturally related to maximizing the quotient of the Weil-Petersson and Teichmüller metrics

$$
\Sigma_{\mathrm{F}}^{2}=\frac{2}{3} \cdot \sup _{(g, X, q)}\left(\frac{\|q\|_{T}}{\|q\|_{\mathrm{WP}}}\right)^{2}
$$

where the supremum is taken over all genera $g \geqslant 2$, Riemann surfaces $X \in \mathcal{T}_{g}$ and quadratic differentials $q \in Q(X)$. Here, we normalize the hyperbolic area of a Riemann surface to be 1 so that the above quotient is invariant under taking finite covers. See [1, Section 7] for details.

In contrast, Theorem 1.4 can be viewed as an attempt to study extremals directly. Inspired by an analogy with a problem in superconductivity in the Euclidean setting, Hedenmalm [9] suggested that extremals satisfy much stronger statistical properties. Understanding the fine structure of extremals would be a major advance in the field. For a possible approach, see the recent work of A. Wennman [29].

In a companion paper [14, joint with I. Kayumov, we show that the equality of universal characteristics remains valid if one takes the supremum over the Bloch unit ball:

$$
\Sigma_{\mathcal{B}}^{2}:=\sup _{\|b\|_{\mathcal{B}} \leqslant 1} \sigma^{2}(b)=\sup _{\|b\|_{\mathcal{B}} \leqslant 1} C_{\mathrm{LIL}}^{2}(b)=\lim _{\tau \rightarrow 0} \frac{1}{|\tau|^{2} / 4} \cdot \sup _{\|b\|_{\mathcal{B}} \leqslant 1} \beta_{b}(\tau) .
$$

In addition, we also give the estimate $\Sigma_{\mathcal{B}}^{2}<\min \left(0.9, \Sigma^{2}\right)$.

Notation. Let $\rho_{*}(z)=\frac{2}{|z|^{2}-1}$ be the density of the hyperbolic metric on the exterior unit disk $\mathbb{D}^{*}$ and $\rho_{\mathbb{H}}(z)=1 / y$ be the corresponding density in the upper half-plane. To compare quantities, we use $A \gtrsim B$ to denote $A>C \cdot B$ for some constant $C>0$. The notation $f f(t) g(t) d t$ denotes the average value of the function $f(t)$ with respect to the measure $g(t) d t$. 


\section{BACKGROUND IN PROBABILITY}

In this section, we discuss martingale analogues of the characteristics of conformal maps mentioned in the introduction. We show that they are controlled by the local variation.

2.1. Martingales and square functions. Let $p \geqslant 2$ be an integer, $\mathcal{D}_{k}$ be the collection of $p$-adic intervals $\left[j \cdot p^{-k},(j+1) \cdot p^{-k}\right]$ contained in $[0,1]$, and $\mathcal{M}_{k}$ be the $\sigma$-algebra generated by $\mathcal{D}_{k}$. A (complex-valued) p-adic martingale $X$ on $[0,1]$ is a sequence of functions $\left\{X_{k}\right\}_{k=0}^{\infty}$ such that

(i) $X_{k}$ is measurable with respect to $\mathcal{M}_{k}$,

(ii) $\mathbb{E}\left(X_{k} \mid \mathcal{M}_{k-1}\right)=X_{k-1}$.

We typically view $X$ as a function from $\bigcup_{k=0}^{\infty} \mathcal{D}_{k}$ to the complex numbers which satisfies the averaging property

$$
X_{I}=\frac{1}{p} \sum_{i=1}^{p} X_{I_{i}},
$$

where the sum ranges over the $p$-adic children of $I$. For a point $x \in[0,1]$, let $I^{\langle k\rangle}(x) \in \mathcal{D}_{k}$ denote the $p$-adic interval of length $p^{-k}$ containing $x$, and $\Delta^{\langle k\rangle}(x)=X_{I^{\langle k\rangle}(x)}-X_{I^{\langle k-1\rangle}(x)}$ be the jump at step $k$. The $p$-adic square function is given by

$$
\langle X\rangle_{n}:=\sum_{k=1}^{n}\left|\Delta^{\langle k\rangle}(x)\right|^{2} .
$$

We say that a martingale has bounded increments if $\left|\Delta^{\langle k\rangle}(x)\right|<C$ for all $x \in[0,1]$ and $k \geqslant 1$. For such martingales, we consider:

- The asymptotic variance

$$
\begin{aligned}
\sigma^{2}(X) & =\limsup _{n \rightarrow \infty} \frac{1}{n} \int_{0}^{1}\left|X_{n}(x)\right|^{2} d x \\
& =\limsup _{n \rightarrow \infty} \frac{1}{n} \int_{0}^{1}\langle X\rangle_{n} d x .
\end{aligned}
$$

(The equality follows from the orthogonality of the jumps.)

- The LIL constant

$$
C_{\mathrm{LIL}}(X)=\operatorname{ess~sup}_{x \in[0,1]}\left\{\limsup _{n \rightarrow \infty} \frac{\left|X_{n}(x)\right|}{\sqrt{n \log \log n}}\right\} .
$$

- The integral means spectrum

$$
\beta_{X}(\tau)=\limsup _{n \rightarrow \infty} \frac{1}{n} \cdot \log \int_{0}^{1}\left|e^{\tau X_{n}(x)}\right| d x, \quad \tau \in \mathbb{C} .
$$


2.2. Local variance. For a $p$-adic interval $I$, we define the local variance of $X$ at $I$ as

$$
\operatorname{Var}_{I} X=\operatorname{Var}_{p, I} X=\frac{1}{p} \sum_{i=1}^{p}\left|X_{I_{i}}-X_{I}\right|^{2} .
$$

More generally, we can consider

$$
\operatorname{Var}_{I}^{n} X=\frac{1}{n}\left[\frac{1}{p^{n}} \sum_{i=1}^{p^{n}}\left|X_{I_{i}}-X_{I}\right|^{2}\right]
$$

where we sum over all $p$-adic grandchildren of $I$ of length $p^{-n}|I|$. Polarizing, we obtain the notion of local covariance

$$
\operatorname{Var}_{I}^{n}(X, Y)=\frac{1}{n}\left[\frac{1}{p^{n}} \sum_{i=1}^{p^{n}}\left(X_{I_{i}}-X_{I}\right) \overline{\left(Y_{I_{i}}-Y_{I}\right)}\right]
$$

of two $p$-adic martingales $X$ and $Y$. Our aim is to show that the local variance controls the above characteristics:

Theorem 2.1. Suppose $S$ is a real-valued martingale with bounded increments. Let $m=\inf _{I} \operatorname{Var}_{I} S$ and $M=\sup _{I} \operatorname{Var}_{I} S$. Then,

(i) For a.e. $x \in[0,1]$,

$$
m \leqslant \liminf \frac{\langle S\rangle_{n}}{n} \leqslant \limsup \frac{\langle S\rangle_{n}}{n} \leqslant M
$$

(ii) $m \leqslant \sigma^{2}(S) \leqslant M$,

(iii) $m \leqslant(1 / 2) \cdot C_{\text {LIL }}^{2}(S) \leqslant M$,

(iv) For $t \in \mathbb{R}$,

$$
m \leqslant \liminf _{t \rightarrow 0} \frac{\beta_{S}(t)}{t^{2} / 2} \leqslant \limsup _{t \rightarrow 0} \frac{\beta_{S}(t)}{t^{2} / 2} \leqslant M .
$$

To evaluate the LIL constant of a martingale, we use a result of W. Stout [27], which is stated explicitly in the form below in [20, Theorem 2.6]:

Lemma 2.2 (Stout). If $S_{n}$ is a real-valued martingale with bounded increments, then

$$
\limsup _{n \rightarrow \infty} \frac{\left|S_{n}(x)\right|}{\sqrt{2\langle S\rangle_{n} \log \log \langle S\rangle_{n}}}=1
$$

almost surely on the set $\left\{x:\langle S\rangle_{\infty}=\infty\right\}$ where $\langle S\rangle_{\infty}=\lim _{n \rightarrow \infty}\langle S\rangle_{n}$.

Proof of Theorem 2.1. (i) Consider the auxiliary martingale $T$ with $T_{[0,1]}=$ 0 and jumps

$$
T_{I_{i}}-T_{I}:=\left|S_{I_{i}}-S_{I}\right|^{2}-\frac{1}{p} \sum_{j=1}^{p}\left|S_{I_{j}}-S_{I}\right|^{2},
$$


where $I_{1}, I_{2}, \ldots, I_{p}$ are the $p$-adic children of $I$. Note that $T$ has bounded increments since $S$ does. Applying Lemma 2.2 to the martingale $T$, we see that

$$
T_{n}(x)=\mathcal{O}(\sqrt{n \log \log n})=o(n), \quad \text { for a.e. } x \in[0,1] .
$$

In particular,

$$
\frac{\langle S\rangle_{n}}{n}=\frac{1}{n} \sum_{k=1}^{n} \operatorname{Var}_{I^{\langle k\rangle}(x)} S+o(1), \quad \text { for a.e. } x \in[0,1] .
$$

The rest is easy: (ii) is trivial, (iii) follows from (i) by Stout's lemma, while (iv) follows from the expansion

$$
\frac{1}{p} \sum_{j=1}^{p} \exp \left(t\left(S_{I_{j}}-S_{I}\right)\right)=1+\frac{t^{2}}{2}\left(\frac{1}{p} \sum_{j=1}^{p}\left|S_{I_{j}}-S_{I}\right|^{2}\right)+\mathcal{O}\left(t^{3}\right) .
$$

This proof is complete.

The same argument shows:

Lemma 2.3. If two real-valued martingales $S_{1}, S_{2}$ satisfy $\operatorname{Var}_{I} S_{1}=\operatorname{Var}_{I} S_{2}$ for all I, then they have the same LIL constant. More generally,

$$
(1 / 2)\left|C_{\mathrm{LIL}}^{2}\left(S_{1}\right)-C_{\mathrm{LIL}}^{2}\left(S_{2}\right)\right| \leqslant \sup _{I}\left|\operatorname{Var}_{I} S_{1}-\operatorname{Var}_{I} S_{2}\right|
$$

The above lemma also holds for the other characteristics discussed in Theorem 2.1.

2.3. Some useful facts. For future reference, we record two martingale estimates. Assume for simplicity that $S$ is a real-valued dyadic martingale with $S_{[0,1]}=0$ and $\left|\Delta^{\langle k\rangle}(x)\right| \leqslant 1$. The sub-Gaussian estimate says that

$$
\left|\left\{x \in[0,1]:\left|S_{n}\right|>t\right\}\right| \leqslant e^{-c t^{2} / n}
$$

for some $c>0$. The sub-Gaussian estimate is a consequence of a more general statement, see [20, Proposition 2.7]. Another proof is given in [8]. Integrating (2.8), we obtain bounds for the moments

$$
\frac{1}{\Gamma(p+1)} \int_{0}^{1}\left|S_{n}\right|^{2 p} d x \leqslant(C n)^{p}, \quad p \geqslant 0 .
$$




\section{Bloch martingales}

In this section, we review Makarov's construction which associates a dyadic martingale to a Bloch function. For convenience, we work in the upper half-plane where the computations are slightly simpler. Therefore, let us imagine that $b$ is a holomorphic function on $\mathbb{H}$ with

$$
\|b\|_{\mathcal{B}(\mathbb{H})}=\sup _{z \in \mathbb{H}} 2 y \cdot\left|b^{\prime}(z)\right| \leqslant 1 .
$$

Here, we assume that $b$ lies in the Bloch unit ball in order to not have to write the Bloch norm all the time.

A dyadic interval $I=\left[x_{1}, x_{2}\right] \subset[0,1]$ defines a 1 -box

$$
\square_{I}=\left\{w: \operatorname{Re} w \in\left[x_{1}, x_{2}\right], \operatorname{Im} w \in\left[\left(x_{2}-x_{1}\right) / 2, x_{2}-x_{1}\right]\right\}
$$

in the upper half-plane. The $n$-box $\square_{I}^{n}$ is defined as the union of 1-boxes associated to $I$ and to all dyadic intervals contained in $I$ of length at least $2^{-n+1}|I|$. For instance,

$$
\square_{[0,1]}^{n}=\left\{w: \operatorname{Re} w \in[0,1], \operatorname{Im} w \in\left[2^{-n}, 1\right]\right\} .
$$

We use $z_{I}=\left(x_{1}+x_{2}\right) / 2+\left(x_{2}-x_{1}\right) i$ to denote the midpoint of the top edge of $\square_{I}$. Following Makarov [20], to the Bloch function $b$ we associate the complex-valued dyadic martingale $B$ given by

$$
B_{I}=\lim _{y \rightarrow 0^{+}} \frac{1}{|I|} \int_{I} b(x+i y) d x .
$$

Makarov showed that the above limit exists and satisfies

$$
\left|b\left(z_{I}\right)-B_{I}\right|=\mathcal{O}(1)
$$

In particular,

$$
\left|B_{I}-B_{J}\right| \leqslant C
$$

whenever $I, J$ are adjacent dyadic intervals of the same size. This is stronger than simply saying that $B$ has bounded increments because $I, J$ may have different parents. Makarov [20] observed that the converse also holds: if a dyadic martingale on $[0,1]$ satisfies the above property, it comes from some Bloch function $b(z)$. It is therefore natural to refer to martingales satisfying (3.4) as Bloch martingales.

One may view dyadic martingales abstractly, defined on the dyadic tree. The notion of a Bloch martingale, however, requires an identification of the dyadic tree with $[0,1]$. One useful fact to keep in mind is: 
Lemma 3.1 (Transmutation principle). For an abstract dyadic martingale with bounded increments, there is an embedding to $[0,1]$ so that it is Bloch.

Sketch of proof. Let $X$ be a dyadic martingale on $[0,1]$ with increments $\left|\Delta^{\langle k\rangle}(x)\right| \leqslant C$. We claim that by flipping the branches at a countable set of dyadic intervals, we can turn $X$ in a dyadic martingale satisfying (3.4) with the same constant $C$. The proof proceeds by induction. Suppose the jump discontinuities of $X_{k}$ (viewed as a function on $[0,1]$ ) at the dyadic points $j / 2^{k}, j=0,1,2, \ldots, 2^{k}$ are bounded by $C$. Let us examine $X_{k+1}$. At the "new" dyadic points $j / 2^{k+1}$ with $j$ odd, the jumps are bounded by $C$ by the assumption on the increments, so one has to only worry about the old dyadic points. It is easy to see that by flipping dyadic intervals of length $1 / 2^{k+1}$, one can make sure that these jumps do not stack up.

The transmutation principle allows one to prove general estimates for dyadic martingales such as 2.9 by working analytically in the realm of Bloch functions.

We define the asymptotic variance, LIL constant and integral means of a Bloch function $b \in \mathcal{B}(\mathbb{H})$ as

$$
\frac{1}{\log 2} \cdot \sigma^{2}(B), \quad \frac{1}{\log 2} \cdot C_{\mathrm{LIL}}^{2}(B), \quad \frac{1}{\log 2} \cdot \beta_{B}(t),
$$

respectively. The factor $\chi=\log 2$ comes from the height of the boxes in the dyadic grid (as measured in the hyperbolic metric). It plays the role of the Lyapunov exponent, cf. [22, Theorem 2.7]. If one instead works with the $p$-adic grid, then the normalizing factor would be $\chi=\log p$. As a consequence of Theorem 3.2 below, the definition of the asymptotic variance can be expressed in function-theoretic terms as

$$
\begin{aligned}
\sigma_{[0,1]}^{2}(b) & =\limsup _{y \rightarrow 0^{+}} \frac{1}{|\log y|} \int_{0}^{1}|b(x+i y)|^{2} d x, \\
& =\limsup _{h \rightarrow 0^{+}} \frac{1}{|\log h|} \int_{h}^{1} \int_{0}^{1}\left|\frac{2 b^{\prime}(x+i y)}{\rho_{\mathbb{H}}}\right|^{2} \frac{|d z|^{2}}{y} .
\end{aligned}
$$

More generally, in [22, Section 6], McMullen showed that one can compute the asymptotic variance by examining Cèsaro averages of integral means that involve higher order derivatives. 


\subsection{A formula for the local variance.}

Theorem 3.2. Suppose $I \subset[0,1]$ is a dyadic interval and $I_{1}, I_{2}, \ldots, I_{2^{n}}$ are its dyadic grandchildren of length $2^{-n}|I|$. Then,

$$
\begin{aligned}
\frac{1}{\log 2} \cdot \operatorname{Var}_{2, I}^{n} B & =\int_{\operatorname{bot}\left(\square_{I}^{n}\right)}\left|b(z)-B_{I}\right|^{2} d x+\mathcal{O}\left(\|b\|_{\mathcal{B}}^{2} / \sqrt{n}\right) \\
& =f_{\square_{I}^{n}}\left|\frac{2 b^{\prime}}{\rho_{\mathbb{H}}}(z)\right|^{2} \frac{|d z|^{2}}{y}+\mathcal{O}\left(\|b\|_{\mathcal{B}}^{2} / \sqrt{n}\right),
\end{aligned}
$$

where in (3.7), we integrate over the bottom side of $\square_{I}^{n}$. Furthermore, we have the "complexification" relations

$$
\operatorname{Var}_{2, I}^{n}(\operatorname{Re} B)=\frac{1}{2} \cdot \operatorname{Var}_{2, I}^{n} B+\mathcal{O}\left(\|b\|_{\mathcal{B}}^{2} / \sqrt{n}\right)
$$

and

$$
\operatorname{Var}_{2, I}^{n}(\operatorname{Re} B, \operatorname{Im} B)=\mathcal{O}\left(\|b\|_{\mathcal{B}}^{2} / \sqrt{n}\right)
$$

Proof. Due to scale invariance, we only need to consider the case when $I=[0,1]$ and $\|b\|_{\mathcal{B}(\mathbb{H})}=1$. We first make some preliminary observations. Since $B$ has bounded jumps,

$$
\langle B\rangle_{n} \lesssim n \quad \text { and } \quad \frac{1}{2^{n}} \sum_{j=1}^{2^{n}}\left|B_{I_{j}}-B_{I}\right|^{2} \lesssim n .
$$

The Cauchy-Schwarz inequality gives

$$
\frac{1}{2^{n}} \sum_{j=1}^{2^{n}}\left|B_{I_{j}}-B_{I}\right| \lesssim \sqrt{n}
$$

The Bloch property implies

$$
\int_{\operatorname{bot}\left(\square_{I}^{n}\right)}\left|b(z)-b\left(z_{I}\right)\right| d s \lesssim \sqrt{n}
$$

and

$$
\left|\int_{\operatorname{bot}\left(\square_{I}^{n}\right)}\right| b(z)-\left.b\left(z_{I}\right)\right|^{2} d s-\frac{1}{2^{n}} \sum_{j=1}^{2^{n}}\left|B_{I_{j}}-B_{I}\right|^{2} \mid \lesssim \sqrt{n} .
$$

Following [23], we apply Green's identity

$$
\int_{\Omega}(u \Delta v-v \Delta u) d x d y=\int_{\partial \Omega}\left(u \cdot \partial_{\mathbf{n}} v-v \cdot \partial_{\mathbf{n}} u\right) d s
$$

where $\partial_{\mathbf{n}}$ refers to differentiation with respect to the outward unit normal vector and $d s$ denotes integration with respect to arc length. The choice

$$
\Omega=\square_{I}^{n}, \quad u=y, \quad v=\left|b(z)-b\left(z_{I}\right)\right|^{2}
$$


yields

$$
\begin{aligned}
& \int_{\square_{I}^{n}} y \cdot\left|2 b^{\prime}(z)\right|^{2} d x d y=\int_{\square_{I}^{n}} y \cdot \Delta\left|b(z)-b\left(z_{I}\right)\right|^{2} d x d y \\
& \quad=-\int_{\partial \square_{I}^{n}} \partial_{\mathbf{n}} y \cdot\left|b(z)-b_{I}(z)\right|^{2} d s+\int_{\partial \square_{I}^{n}} y \cdot \partial_{\mathbf{n}}\left|b(z)-b\left(z_{I}\right)\right|^{2} d s .
\end{aligned}
$$

It is evident that

$$
-\int_{\partial \square_{I}^{n}} \partial_{\mathbf{n}} y \cdot\left|b(z)-b\left(z_{I}\right)\right|^{2} d s=\int_{\operatorname{bot}\left(\square_{I}^{n}\right)}\left|b(z)-b\left(z_{I}\right)\right|^{2} d s-\mathcal{O}(1) .
$$

For the error term,

$$
\int_{\partial \square_{I}^{n}} y \cdot \partial_{\mathbf{n}}\left|b(z)-b\left(z_{I}\right)\right|^{2} d s \lesssim \int_{\partial \square_{I}^{n}}\left|b(z)-b\left(z_{I}\right)\right| d s .
$$

From the definition of a Bloch function (3.1), the integral of $\left|b(z)-b\left(z_{I}\right)\right|$ over the top, left and right sides of $\partial \square_{I}^{n}$ is $\mathcal{O}(1)$, while according to $(3.12)$, the integral over the bottom side is $\mathcal{O}(\sqrt{n})$. Summarizing, we see that

$$
f_{\square_{I}^{n}}\left|\frac{2 b^{\prime}(z)}{\rho_{\mathbb{H}}}\right|^{2} \frac{|d z|^{2}}{y}=\frac{1}{n \log 2} \int_{\operatorname{bot}\left(\square_{I}^{n}\right)}\left|b(z)-b\left(z_{I}\right)\right|^{2} d s+\mathcal{O}(1 / \sqrt{n}) .
$$

Combining with 3.13 gives another error of $\mathcal{O}(1 / \sqrt{n})$ and proves 3.8 .

Repeating the above argument with $v=\left|\operatorname{Re} b-\operatorname{Re} b\left(z_{I}\right)\right|^{2}$ gives

$$
\begin{aligned}
\frac{\operatorname{Var}_{2, I}^{n}(\operatorname{Re} B)}{\log 2} & =\int_{\operatorname{bot}\left(\square_{I}^{n}\right)}\left|\operatorname{Re} b(z)-\operatorname{Re} B_{I}\right|^{2} d x+\mathcal{O}\left(\|b\|_{\mathcal{B}}^{2} / \sqrt{n}\right) \\
& =\frac{1}{2} f_{\square_{I}^{n}}\left|\frac{2 \nabla(\operatorname{Re} b)}{\rho_{\mathbb{H}}}(z)\right|^{2} \frac{|d z|^{2}}{y}+\mathcal{O}\left(\|b\|_{\mathcal{B}}^{2} / \sqrt{n}\right),
\end{aligned}
$$

which proves $(3.9)$ since $\left|b^{\prime}\right|=|\nabla(\operatorname{Re} b)|$. Finally $(3.10)$ follows from polarization and the identity $\nabla(\operatorname{Re} b) \cdot \nabla(\operatorname{Im} b)=0$ which is a consequence of the Cauchy-Riemann relations.

\subsection{Complexification.}

Theorem 3.3 (Complexification). For any Bloch function $b \in \mathcal{B}(\mathbb{H})$,

$$
\sigma^{2}(\operatorname{Re} b)=(1 / 2) \cdot \sigma^{2}(b), \quad C_{\mathrm{LIL}}(\operatorname{Re} b)=C_{\mathrm{LIL}}(b) .
$$

Proof. The first statement is an immediate consequence of 3.9 ). For the second statement, note that the function $\theta \rightarrow C_{\mathrm{LIL}}\left(\operatorname{Re} e^{i \theta} B\right)$ is continuous and

$$
C_{\mathrm{LIL}}(B)=\sup _{\theta \in[0,2 \pi)} C_{\mathrm{LIL}}\left(\operatorname{Re} e^{i \theta} B\right)
$$


We must therefore show $C_{\mathrm{LIL}}\left(\operatorname{Re} e^{i \theta} B\right) \leqslant C_{\mathrm{LIL}}(\operatorname{Re} B)$, for any $\theta \in[0,2 \pi)$. However, if view $\operatorname{Re} B$ and $\operatorname{Re} e^{i \theta} B$ as $p$-adic martingales with $p=2^{n}$ large, then by $(3.9)$, their local variances are approximately equal. The assertion now follows from Lemma 2.3 .

Remark. For lacunary series, the equality $C_{\mathrm{LIL}}(\operatorname{Re} b)=C_{\mathrm{LIL}}(b)$ goes back to the 1959 work of M. Weiss [28].

\section{The Box Lemma}

Let $\mathbf{H}_{k}$ denote the class of conformal maps $f: \mathbb{H} \rightarrow \mathbb{C}$ which admit a $k$-quasiconformal extension to the plane and fix the points $0,1, \infty$. For $f \in \mathbf{H}_{k}$, we denote the associated Bloch function by $b_{f}=\log f^{\prime} \in \mathcal{B}(\mathbb{H})$. It is not difficult to show that the expressions

$$
\sup _{f \in \mathbf{H}_{k}} \sigma^{2}\left(b_{f}\right), \quad \sup _{f \in \mathbf{H}_{k}} C_{\text {LIL }}^{2}\left(b_{f}\right), \quad \sup _{f \in \mathbf{H}_{k}} \beta_{b_{f}}(t),
$$

coincide with their analogues for the class $\mathbf{S}_{k}$ from the introduction. The proof of Theorem 1.2 is now completed by the Box Lemma from [13] which describes the average non-linearity $n_{f}:=f^{\prime \prime} / f^{\prime}=\left(\log f^{\prime}\right)^{\prime}$ of conformal mappings:

Lemma 4.1. (i) Fix $0<k<1$. Given $\varepsilon>0$, there exists $n \geqslant 1$ sufficiently large so that for any $n$-box $\square_{I}^{n} \subset \mathbb{H}$ and any conformal map $f \in \mathbf{H}_{k}$,

$$
f_{\square_{I}^{n}}\left|\frac{2 n_{f}}{\rho_{\mathbb{H}}}(z)\right|^{2} \frac{|d z|^{2}}{y}<\Sigma^{2}(k)+\varepsilon .
$$

(ii) Conversely, for any $\varepsilon>0$, there exists a conformal map $f \in \mathbf{H}_{k}$, whose dilatation dil. $f:=\bar{\partial} f / \partial f$ is periodic with respect to the $2^{n}$-adic grid for some $n \geqslant 1$, and which satisfies

$$
f_{\square_{I}^{n}}\left|\frac{2 n_{f}}{\rho_{\mathbb{H}}}(z)\right|^{2} \frac{|d z|^{2}}{y}>\Sigma^{2}(k)-\varepsilon,
$$

on every $n$-box $\square_{I}^{n}$.

Remark. The proof given in [13] forces us to restrict our attention to classes of conformal maps with bounded distortion. It would be interesting to know if a variant of the box lemma holds for all conformal maps with $\lim _{k \rightarrow 1^{-}} \Sigma^{2}(k)$ in place of $\Sigma^{2}(k)$. 
In view of Theorems 2.1 and 3.2 , (i) gives the upper bound in Theorem 1.2 , while (ii) gives the lower bound. The notion of periodic Beltrami coefficients will be discussed below in Section 4.1. In order to state the infinitesimal version of the box lemma, note that the formula for the Beurling transform (1.6) may not converge if $\mu$ is not compactly supported. Therefore, we are obliged to work with a modified Beurling transform

$$
\mathcal{S}^{\#} \mu(z)=-\frac{1}{\pi} \int_{\mathbb{H}} \mu(\zeta)\left[\frac{1}{(\zeta-z)^{2}}-\frac{1}{\zeta(\zeta-1)}\right]|d \zeta|^{2} .
$$

However, the formula for the derivative remains the same:

$$
\text { " }(\mathcal{S} \mu)^{\prime}(z) ":=\left(\mathcal{S}^{\#} \mu\right)^{\prime}(z)=-\frac{2}{\pi} \int_{\mathbb{H}} \frac{\mu(\zeta)}{(\zeta-z)^{3}}|d \zeta|^{2} .
$$

In [13, the infinitesimal analogue of the box lemma was proved with a quantitative relation between the box size and the error term:

Lemma 4.2. (i) For any Beltrami coefficient $\mu$ with $|\mu| \leqslant \chi_{\overline{\mathbb{H}}}$ and $n$-box $\square_{I}^{n} \subset \mathbb{H}$,

$$
f_{\square_{I}^{n}}\left|\frac{2(\mathcal{S} \mu)^{\prime}}{\rho_{\mathbb{H}}}(z)\right|^{2} \frac{|d z|^{2}}{y}<\Sigma^{2}+C / n .
$$

(ii) Conversely, for $n \geqslant 1$, there exists a Beltrami coefficient $\mu$, periodic with respect to the $2^{n}$-adic grid, which satisfies

$$
f_{\square_{I}^{n}}\left|\frac{2(\mathcal{S} \mu)^{\prime}}{\rho_{\mathbb{H}}}(z)\right|^{2} \frac{|d z|^{2}}{y}>\Sigma^{2}-C / n
$$

on every $n$-box $\square_{I}^{n}$.

The quantitative estimate will be exploited in Section 5

4.1. Periodic Beltrami coefficients. Given two intervals $I, J \subset \mathbb{R}$, let $L_{I, J}(z)=A z+B$ be the unique linear map with $A>0, B \in \mathbb{R}$ that maps $I$ to $J$. For a box $\square$, we denote its reflection in the real line by $\bar{\square}$. Suppose $\mu$ is a Beltrami coefficient supported on the lower half-plane. We say that $\mu$ is periodic (with respect to the dyadic grid) if for any two dyadic intervals $I, J \subset \mathbb{R}$ with $|I|,|J| \leqslant 1,\left.\mu\right|_{\square_{I}}=L_{I, J}^{*}\left(\left.\mu\right|_{\square_{J}}\right)$. We typically assume that $\mu$ is supported on the strip

$$
\{w:-1<\operatorname{Im} w<0\},
$$

in order for $\mu$ to be invariant under translation by 1 . In this case, $\mu$ descends to a Beltrami coefficient on the disk via the exponential mapping, which is 
eventually-invariant under $z \rightarrow z^{2}$. The notion of a Beltrami coefficient periodic with respect to the $p$-adic grid is defined similarly.

Before continuing further, we define a dyadic box in the unit disk to be the image of $\square_{I},|I| \leqslant 1$, under the exponential mapping $\xi(w)=\exp (2 \pi i w)$. Reflecting in the unit circle, we obtain a dyadic box in the exterior unit disk. Note that these boxes are not geometric rectangles, nor do they tile $\mathbb{D}$ or $\mathbb{D}^{*}$ completely.

4.2. Dynamical Beltrami coefficients. We now consider two classes of dynamical Beltrami coefficients on the unit disk that naturally arise in complex dynamics and Teichmüller theory:

- $M_{\mathrm{B}}=\bigcup_{f} M_{f}(\mathbb{D})$ consists of Beltrami coefficients that are eventuallyinvariant under some finite Blaschke product

$$
f(z)=z \prod_{i=1}^{d-1} \frac{z-a_{i}}{1-\overline{a_{i}} z},
$$

i.e. Beltrami coefficients which satisfy $f^{*} \mu=\mu$ in some open neighbourhood of the unit circle.

- $M_{\mathrm{F}}=\bigcup_{\Gamma} M_{\Gamma}(\mathbb{D})$ consists of Beltrami coefficients that are invariant under some co-compact Fuchsian group $\Gamma$, i.e. $\gamma^{*} \mu=\mu$ for all $\gamma \in \Gamma$.

Suppose $\mu$ belongs to one of the two classes of Beltrami coefficients above, with $\|\mu\|_{\infty}<1$. We view $f=w^{\mu}$ as a conformal map of the exterior unit disk. From the construction, the image of the unit circle $f\left(\mathbb{S}^{1}\right)$ is a Julia set or a limit set of a quasi-Fuchsian group. Using the ergodicity of the geodesic flow on the unit tangent bundle $T_{1} X$ (Fuchsian case) or Riemann surface lamination $\hat{X}_{B}$ (Blaschke case), it is not hard to show that for any $\varepsilon>0$, there exists $n_{0}$ sufficiently large,

$$
\sigma^{2}\left(\log f^{\prime}\right)-\varepsilon<f_{\square_{I}^{n}}\left|\frac{2 n_{f}}{\rho_{*}}(z)\right|^{2} \rho_{*}|d z|^{2}<\sigma^{2}\left(\log f^{\prime}\right)+\varepsilon
$$

for any $n$-box $\square_{I}^{n} \subset \mathbb{D}^{*}$ with $n \geqslant n_{0}$. Applying Theorems 2.1 and 3.2 shows that Theorem 1.1 holds for conformal maps $f=w^{\mu}$ with $\mu \in M_{\mathrm{B}}$ or $M_{\mathrm{F}}$. More generally, one can prove 4.7 for conformal maps to simply-connected domains bounded by Jordan repellers, see [1, Section 8] for a definition. The reader interested in working out the details can consult [22]. 


\section{Applications to integral means}

In this section, we use martingale techniques to study the rescaled integral means spectrum

$$
B_{*}(\tau)=\lim _{k \rightarrow 0} B_{k}(\tau / k)=\sup _{|\mu| \leqslant \chi_{\overline{\mathbb{H}}}} \beta_{\mathcal{S}^{\#} \mu}(\tau),
$$

near $\tau=0$. Let $B$ be the dyadic martingale associated to the Bloch function $b=\mathcal{S}^{\#} \mu,|\mu| \leqslant \chi_{\overline{\mathbb{H}}}$ and $S=\operatorname{Re} B$ be its real part.

Since $B_{*}(\tau)$ is radially invariant, it suffices to estimate $\beta_{S}(t)$ with $t \in \mathbb{R}$. We view $S$ as a $p$-adic martingale with $p=2^{n}$, where the parameter $n$ will be chosen momentarily. Suppose $I$ is a $2^{n}$-adic interval and $I_{1}, I_{2}, \ldots, I_{2^{n}}$ are its $2^{n}$-adic children. Letting $\Delta_{j}=S_{I_{j}}-S_{I}$,

$$
\begin{aligned}
\frac{1}{2^{n}} \sum_{j=1}^{2^{n}} \exp \left(t \Delta_{j}\right) & =1+\frac{t^{2}}{2}\left(\frac{1}{2^{n}} \sum_{j=1}^{2^{n}} \Delta_{j}^{2}\right)+\sum_{k \geqslant 3} \frac{t^{k}}{k !}\left(\frac{1}{2^{n}} \sum_{j=1}^{2^{n}} \Delta_{j}^{k}\right), \\
& =1+\frac{t^{2}}{2} \operatorname{Var}_{2^{n}, I} S+\mathcal{O}\left(\sum_{k \geqslant 3} \frac{t^{k}}{k !} \cdot(C n)^{k / 2}\right) .
\end{aligned}
$$

Above, we used 2.9 to estimate the remainder term. Making use of Lemma $4.2(\mathrm{i})$, we see that the above expression is bounded by

$$
\leqslant 1+\frac{n t^{2}}{2}\left(\frac{\Sigma^{2} \log 2}{2}+\mathcal{O}(1 / \sqrt{n})+\mathcal{O}\left(t n^{1 / 2}\right)+\ldots\right)
$$

Note that in order to use martingale techniques, we had to downgrade the box estimate with $\Sigma^{2} / 2+C / n$ to the variance bound $\operatorname{Var}_{2^{n}, I} S /(n \log 2) \leqslant$ $\Sigma^{2} / 2+C / \sqrt{n}$, cf. Theorem 3.2 . Hence,

$$
\frac{1}{n \log 2} \log \left[\frac{1}{2^{n}} \sum_{j=1}^{2^{n}} \exp \left(t \Delta_{j}\right)\right] \leqslant \frac{t^{2}}{2}\left(\frac{\Sigma^{2}}{2}+\mathcal{O}(1 / \sqrt{n})+\mathcal{O}\left(t n^{1 / 2}\right)+\ldots\right) .
$$

Taking $n=\left\lfloor t^{-1}\right\rfloor$ leads to the estimate

$$
B_{*}(t) \leqslant \Sigma^{2} t^{2} / 4+\mathcal{O}\left(|t|^{5 / 2}\right) .
$$

By using Lemma 4.2(ii), one can also use the above reasoning to obtain a lower bound for integral means spectrum, thus showing that (5.1) is an equality. As mentioned in the introduction, if one avoids martingales, one can obtain a remainder term better than $\mathcal{O}\left(|t|^{5 / 2}\right)$.

Recently, Hedenmalm [8] proved the beautiful estimate $B_{*}(\tau) \leqslant|\tau|^{2} / 4$ for all $\tau \in \mathbb{C}$. While not sharp near the origin, this estimate cannot be 
improved for general values of $\tau$, e.g. $B_{*}(2)=1$ since $B_{k}(2 / k)=1$ for all $0<k<1$.

\section{A central limit theorem}

Suppose $b=\log f^{\prime}$ with $f \in \mathbf{S}_{k}$. Consider the function

$$
\tilde{b}_{r}(\theta):=\frac{b\left(r e^{i \theta}\right)}{\sqrt{|\log (1-r)|}} .
$$

The sub-Gaussian estimate 2.8 shows that most of the integral $\int\left(\operatorname{Re} \tilde{b}_{r}(\theta)\right)^{2} d \theta$ comes from the set

$$
A_{\delta}=\left\{\theta:-1 / \delta<\operatorname{Re} \tilde{b}_{r}(\theta)<1 / \delta\right\},
$$

that is, by making $\delta>0$ small, we can guarantee that

$$
\int_{A_{\delta}^{c}}\left(\operatorname{Re} \tilde{b}_{r}(\theta)\right)^{2} d \theta<\varepsilon
$$

with the estimate being uniform over all functions $b$ of the form above. In this section, we show that if $\sigma^{2}(b, r)$ is close to $\Sigma^{2}(k)$, then the distribution of $\operatorname{Re} \tilde{b}_{r}(\theta)$ is close to a Gaussian of mean 0 and variance $\Sigma^{2}(k) / 2$.

Theorem 6.1. Let $0<k<1$ be fixed. Suppose $f \in \mathbf{S}_{k}$ and $b=\log f^{\prime}$. Given $\varepsilon>0$, there exists a $\delta>0$ such that if $r$ is sufficiently close to 1 and

$$
\sigma^{2}(b, r)=\frac{1}{2 \pi|\log (1-r)|} \int_{|z|=r}|b(z)|^{2}|d z|>\Sigma^{2}(k)-\delta
$$

then for any $t \in \mathbb{R}$,

$$
\left|\mathbb{P}\left(\operatorname{Re} \tilde{b}_{r}(\theta)<t\right)-\mathcal{N}_{0, \Sigma^{2}(k) / 2}(t)\right|<\varepsilon .
$$

The same statement holds with $\operatorname{Im} \tilde{b}_{r}(\theta)$ as well.

Converting to the upper half-plane, let $B$ be the $p$-adic martingale associated to the Bloch function $b=\log f^{\prime}, f \in \mathbf{H}_{k}$ and $S=\operatorname{Re} B$ be its real part. In view of Theorem 3.2 and Lemma 4.1 , we can choose $p=2^{m}$ sufficiently large to guarantee

$$
\begin{gathered}
f_{\square_{I}^{(p)}}\left|\frac{2 b^{\prime}}{\rho_{\mathbb{H}}}(z)\right|^{2} \frac{|d z|^{2}}{y}<\Sigma^{2}(k)+\delta_{1} / 2, \\
\left.\left|\frac{\operatorname{Var}_{p, I} S}{\chi}-\frac{1}{2} f_{\square_{I}^{(p)}}\right| \frac{2 b^{\prime}}{\rho_{\mathbb{H}}}(z)\right|^{2} \frac{|d z|^{2}}{y} \mid<\delta_{1} / 2,
\end{gathered}
$$


for all $p$-adic intervals $I$. Here, $\chi=\log p$ is the Lyapunov exponent of the $p$-adic grid and

$$
\square_{I}^{(p)}=\left\{w: \operatorname{Re} w \in I, \operatorname{Im} w \in\left[p^{-1}|I|,|I|\right]\right\}
$$

denotes the $p$-adic box associated to $I$.

6.1. Characteristic functions. As is standard [6], to prove the central limit theorem, we need to examine the characteristic functions

$$
\varphi_{n}(t)=\mathbb{E} \exp \left(i \cdot \frac{t S_{n}}{\sqrt{n \chi}}\right), \quad t \in \mathbb{R} .
$$

Even though martingale jumps are usually not independent, in extremal situations, one can instead leverage the fact that the local variance is approximately constant. Observe that if $I$ is a $p$-adic interval and $I_{1}, I_{2}, \ldots, I_{p}$ are its children, then for $t$ small,

$$
\frac{1}{p} \sum_{j=1}^{p} e^{i t \Delta_{j}}=1-\frac{t^{2}}{2} \operatorname{Var}_{p, I} S+\mathcal{O}\left(t^{3}\right) .
$$

The above formula shows that if a martingale $S$ has constant local variance, that is if $\operatorname{Var}_{p, I} S / \chi=\sigma^{2}$ for all $p$-adic intervals $I$, then

$$
\varphi_{n}=\left[1-\frac{\sigma^{2} t^{2}}{2 n}+\mathcal{O}\left(\frac{t^{3}}{n^{3 / 2}}\right)\right]^{n}
$$

Fixing $t \in \mathbb{R}$ and taking $n \rightarrow \infty$ yields

$$
\varphi=\lim _{n \rightarrow \infty} \varphi_{n}=\exp \left(-\frac{\sigma^{2} t^{2}}{2}\right)
$$

which is the characteristic function of the Gaussian $\mathcal{N}_{0, \sigma^{2}}$. In this case, continuity properties of characteristic functions (e.g. [6, Theorem 3.3.6]) imply that the distributions of $S_{n} / \sqrt{n \chi}$ converge to $\mathcal{N}_{0, \sigma^{2}}$ as $n \rightarrow \infty$.

More generally, if the local variance is pinched,

$$
\sigma^{2}-\delta_{1} \leqslant \operatorname{Var}_{p, I} S / \chi \leqslant \sigma^{2}+\delta_{1}, \quad \forall I,
$$

then

$$
\varphi_{n}(t)=\left[1-\frac{\sigma_{n}^{2} t^{2}}{2 n}+\mathcal{O}\left(\frac{t^{3}}{n^{3 / 2}}\right)\right]^{n}, \quad \text { with }\left|\sigma_{n}-\sigma^{2}\right| \leqslant \delta_{1} .
$$

Like in the case of constant local variance, we can still conclude that

$$
\left|\mathbb{P}\left(S_{n} / \sqrt{n \chi}<t\right)-\mathcal{N}_{0, \sigma^{2}}(t)\right| \leqslant \varepsilon\left(\delta_{1}\right), \quad \text { for } n \geqslant n_{0}(p, \varepsilon) \text { large, }
$$

with $\varepsilon\left(\delta_{1}\right) \rightarrow 0$ as $\delta_{1} \rightarrow 0$. 
6.2. Allowing bad boxes. For the problem at hand, we must allow a small proportion of $p$-adic intervals $I$ to be bad for which the lower bound in 6.6 ) fails. Set

$$
\square_{[0,1]}^{n}=\left\{w: \operatorname{Re} w \in[0,1], \operatorname{Im} w \in\left[p^{-n}, 1\right]\right\} .
$$

Call a $p$-adic box $\square_{I}^{(p)}$ good if the lower bound in 6.6 holds and bad otherwise. Fix $n \geqslant 1$ and let $\mathscr{E} \subset \square_{[0,1]}^{n}$ denote the union of bad boxes contained in $\square_{[0,1]}^{n}$.

Lemma 6.2. Suppose $S$ is a real-valued p-adic martingale on $[0,1]$ with

$$
\begin{gathered}
\operatorname{Var}_{p, I} S / \chi \leqslant \sigma^{2}+\delta_{1}, \quad \forall I, \\
\operatorname{Var}_{p, I} S / \chi \geqslant \sigma^{2}-\delta_{1}, \quad I \text { good, } \\
f_{\square_{[0,1]}^{n} \chi_{\mathscr{E}} \cdot \frac{|d z|^{2}}{y}} \lesssim \delta_{2}, \quad \mathscr{E}=\bigcup_{I \text { bad }} \square_{I}^{(p)} .
\end{gathered}
$$

Given any $\varepsilon>0$, one can choose the parameters $\delta_{1}$ and $\delta_{2}$ small enough to guarantee

$$
\left|\mathbb{P}\left(S_{n} / \sqrt{n \chi}<t\right)-\mathcal{N}_{0, \sigma^{2}}(t)\right| \leqslant \varepsilon\left(\delta_{1}, \delta_{2}\right), \quad \text { for } n \geqslant n_{0}(p, \varepsilon) \text { large. }
$$

To prove the above lemma, we will use the following elementary identity: if $X$ is a $p$-adic martingale on $[0,1]$, then

$$
\sigma^{2}(X, n) / \chi:=\frac{1}{n \chi} \int_{0}^{1}\left(X_{n}\right)^{2} d x=\frac{X_{[0,1]}^{2}}{n \chi}+f_{\square_{[0,1]}^{n}} \operatorname{Var}_{p, I(z)} X \cdot \frac{|d z|^{2}}{y}
$$

where $I(z)$ is the $p$-adic interval $I$ for which $z \in \square_{I}^{(p)}$. This identity is a simple consequence of the orthogonality of increments $\int_{0}^{1} \Delta_{X}^{\left\langle k_{1}\right\rangle}(x) \Delta_{X}^{\left\langle k_{2}\right\rangle}(x) d x$ for $k_{1} \neq k_{2}$.

Proof. Write $S=S^{\text {good }}+S^{\text {bad }}$ as a sum of two martingales, where the local variance of $S^{\text {good }}$ is close to $\sigma^{2}$ on all intervals while the increments of $S^{\text {bad }}$ are non-zero only on bad intervals. We may form $S^{\text {good }}$ from $S$ by adjusting the jumps on the bad intervals, and defining $S^{\text {bad }}:=S-S^{\text {good }}$ to be the difference. From the construction, it is clear that

$$
\sigma^{2}\left(S^{\text {bad }}, n\right) / \chi=\frac{1}{n \chi} \int_{0}^{1}\left(S_{n}^{\text {bad }}\right)^{2} d x \lesssim \delta_{2} \cdot\left(\sigma^{2}+\delta_{1}\right)
$$

which shows that $S_{n}^{\mathrm{bad}} / \sqrt{n \chi}$ is small outside of a set of small measure. Therefore, the distribution of $S_{n} / \sqrt{n \chi}$ is roughly that of $S_{n}^{\text {good }} / \sqrt{n \chi}$, which we already know to be approximately Gaussian. 
6.3. Conclusion. Having Lemma 6.2 at our disposal, the proof of Theorem 6.1 is almost immediate. We now resume the discussion from the beginning of this section. Recall that $S=\operatorname{Re} B$ is the $p$-adic martingale associated to a Bloch function $b=\log f^{\prime}$ with $f \in \mathbf{H}_{k}$, and $p$ was chosen to guarantee 6.4), 6.5.

Proof of Theorem 6.1. Inspecting (3.7) and making use of 6.4), (6.5) and (6.11), we see that if the inequality

$$
\sigma^{2}(S, n) / \chi=\frac{1}{n \chi} \int_{0}^{1} S_{n}^{2} d x+o(1) \geqslant\left(\Sigma^{2}(k)-\delta\right) / 2
$$

holds for some $0<\delta<\delta_{1}$, then the exceptional set $\mathscr{E}$ defined in the previous section satisfies

$$
f_{\square_{[0,1]}^{n}} \chi_{\mathscr{E}} \cdot \frac{|d z|^{2}}{y} \lesssim \delta / \delta_{1}, \quad \text { with } \sigma^{2}=\Sigma^{2}(k) / 2 .
$$

Since $\delta / \delta_{1}$ can be made arbitrarily small by requesting $\delta$ to be small, Lemma 6.2 is applicable and shows that if $n \geqslant n_{0}$ is sufficiently large, then the distribution of $S_{n} / \sqrt{n \chi}$ is close to that of the Gaussian $\mathcal{N}_{0, \Sigma^{2}(k) / 2}$. Taking advantage of the approximation property of Bloch martingales (3.3), we have proved that the statement

$$
\frac{1}{|\log y|} \int_{0}^{1}|\operatorname{Re} b(x+i y)|^{2} d x \geqslant\left(\Sigma^{2}(k)-\delta\right) / 2, \quad y=p^{-n}
$$

implies that $\operatorname{Re} \tilde{b}_{y}(x):=\operatorname{Re} b(x+i y) / \sqrt{|\log y|} \approx \mathcal{N}_{0, \Sigma^{2}(k) / 2}$ in distribution. For general $y$, not necessarily of the form $p^{-n}$, the Lipschitz property of Bloch functions implies $\operatorname{Re} \tilde{b}_{y}(x) \approx \operatorname{Re} \tilde{b}_{p^{-n}}(x)$, with $n=\left\lfloor\log _{p} 1 / y\right\rfloor$. Finally, to replace $\operatorname{Re} b$ with $b$ in 6.14 , we appeal to the complexification relation (3.9), see also (3.16). This completes the proof.

The proof of Theorem 1.4 is similar except one considers characteristic functions of two variables

$$
\varphi_{n}(s, t)=\mathbb{E} \exp \left(i \cdot \frac{s \operatorname{Re} B+t \operatorname{Im} B}{\sqrt{n \chi}}\right)
$$

and uses the approximate orthogonality 3.10 between $\operatorname{Re} B$ and $\operatorname{Im} B$ to show $\varphi_{n}(s, t) \approx \exp \left(-\frac{\Sigma^{2}(k)\left(s^{2}+t^{2}\right)}{4}\right)$. We leave the details to the reader. 


\section{REFERENCES}

1. K. Astala, O. Ivrii, A. Perälä, I. Prause, Asymptotic variance of the Beurling transform, Geom. Funct. Anal. 25 (2015), no. 6, 1647-1687.

2. K. Astala, T. Iwaniec, G. J. Martin, Elliptic partial differential equations and quasiconformal mappings in the plane, Princeton University Press, 2009.

3. R. Bañuelos, C. N. Moore, Mean growth of Bloch functions and Makarov's law of the iterated logarithm, Proc. Amer. Math. Soc. 112 (1991), 851-854.

4. J. Becker, C. Pommerenke, On the Hausdorff dimension of quasicircles, Ann. Acad. Sci. Fenn. Ser. A I Math. 12 (1987), 329-333.

5. I. Binder, Harmonic measure and rotation spectra of planar domains, preprint, 2008.

6. R. Durrett, Probability: Theory and Examples, Cambridge Series in Statistical and Probabilistic Mathematics, Cambridge University Press, 4th edition, 2010.

7. J. B. Garnett, D. E. Marshall, Harmonic Measure, New Mathematical Monographs 2, Cambridge University Press, 2005.

8. H. Hedenmalm, Bloch functions and asymptotic tail variance, preprint, 2015. arXiv:1509.06630

9. H. Hedenmalm, Bloch functions, asymptotic variance and geometric zero packing, preprint, 2016. arXiv:1602.03358

10. H. Hedenmalm, I. R. Kayumov, On the Makarov law of the iterated logarithm, Proc. Amer. Math. Soc. 135 (2007), 2235-2248.

11. H. Hedenmalm, S. Shimorin, Weighted Bergman spaces and the integral means spectrum of conformal mappings, Duke Math. J. 127 (2005), 341-393.

12. H. Hedenmalm, S. Shimorin, On the universal integral means spectrum of conformal mappings near the origin, Proc. Amer. Math. Soc. 135 (2007), no. 7, 2249-2255.

13. O. Ivrii, Quasicircles of dimension $1+k^{2}$ do not exist, preprint, 2016. arXiv: 1511.07240

14. O. Ivrii, I. R. Kayumov, Makarov's principle for the Bloch unit ball, preprint, 2016. arXiv:1605.00246

15. I. R. Kayumov, The law of the iterated logarithm for locally univalent functions, Ann. Acad. Sci. Fenn. Math. 27 (2002), no. 2, 357-364.

16. R. Kühnau, Möglichst konforme Spiegelung an einer Jordankurve, Jahresber. Deutsch. Math.-Verein. 90 (1988), no. 2, 90-109.

17. T. H. N. Le, M. Zinsmeister, On Minkowski dimension of Jordan curves, Ann. Acad. Sci. Fenn. Math. 39 (2014), no. 2, 787-810.

18. N. G. Makarov, On the distortion of boundary sets under conformal mappings, Proc. London Math. Soc. 51(1985), 369-384.

19. N. G. Makarov, Conformal mapping and Hausdorff measures, Ark. Mat. 25 (1987), no. 1, 41-89.

20. N. G. Makarov, Probability methods in the theory of conformal mappings, (Russian) Algebra i Analiz 1 (1989), no. 1, 3-59; translation in Leningrad Math. J. 1 (1990), no. $1,1-56$.

21. N. G. Makarov, Fine structure of harmonic measure, St. Petersburg Math. J. 10 (1999), no. 2, 217-268.

22. C. T. McMullen, Thermodynamics, dimension and the Weil-Petersson metric, Invent. Math. 173 (2008), no. 2, 365-425.

23. A. Nicolau, Radial behaviour of harmonic Bloch functions and their area function, Indiana Univ. Math. J. 48 (1999), no. 4, 1213-1236.

24. C. Pommerenke, Boundary behaviour of conformal maps, Grundlehren der Mathematischen Wissenschaften 299, Springer-Verlag, 1992.

25. F. Przytycki, M. Urbański, A. Zdunik, Harmonic, Gibbs and Hausdorff measures on repellers for holomorphic maps II, Studia Math. 97 (1991), 189-225.

26. S. Smirnov, Dimension of quasicircles, Acta Math. 205 (2010), no. 1, 189-197. 
27. W. Stout, A martingale analogue of Kolmogorov's law of the iterated logarithm, Z. Wahrsch. Verw. Gebiete. 15 (1970), 279-290.

28. M. Weiss, The law of the iterated logarithm for lacunary trigonometric series, Trans. Amer. Math. Soc. 91 (1959), 444-469.

29. A. Wennman, Discrepancy densities for planar and hyperbolic Zero Packing, preprint, 2016. arXiv:1605.08674

Department of Mathematics and Statistics, University of Helsinki, P.O. Box 68, FIN-00014, Helsinki, Finland

E-mail address: oleg.ivrii@helsinki.fi 